\title{
Synchronization of nonidentical chaotic neural networks with leakage delay and mixed time- varying delays
}

\author{
Qiankun Song ${ }^{1}$ and Jinde $\mathrm{Cao}^{2^{*}}$
}

\author{
* Correspondence: jdcao@seu.edu. \\ cn \\ ${ }^{2}$ Department of Mathematics, \\ Southeast University, Nanjing \\ 210096, China \\ Full list of author information is \\ available at the end of the article
}

\begin{abstract}
In this paper, an integral sliding mode control approach is presented to investigate synchronization of nonidentical chaotic neural networks with discrete and distributed time-varying delays as well as leakage delay. By considering a proper sliding surface and constructing Lyapunov-Krasovskii functional, as well as employing a combination of the free-weighting matrix method, Newton-Leibniz formulation and inequality technique, a sliding mode controller is designed to achieve the asymptotical synchronization of the addressed nonidentical neural networks. Moreover, a sliding mode control law is also synthesized to guarantee the reachability of the specified sliding surface. The provided conditions are expressed in terms of linear matrix inequalities, and are dependent on the discrete and distributed time delays as well as leakage delay. A simulation example is given to verify the theoretical results.
\end{abstract}

Keywords: Synchronization, Chaotic neural network, Leakage delay, Discrete timevarying delays, Distributed time-varying delays 05.45.Xt 05.45.Gg

\section{Introduction}

In the past few years, neural networks have attracted much attention due to the background of a wide range applications such as associative memory, pattern recognition, image processing and model identification [1]. In such applications, the qualitative analysis of the dynamical behaviors is a necessary step for the practical design of neural networks [2].

In hardware implementation, time delays occur due to finite switching speed of the amplifiers and communication time. The existence of time delay may lead to some complex dynamic behaviors such as oscillation, divergence, chaos, instability, or other poor performance of the neural networks [3]. Therefore, the study of dynamical behaviors with consideration of time delays becomes extremely important to manufacture high-quality neural networks [4]. Many results on dynamical behaviors have been reported for delayed neural networks, for example, see [1-10] and references therein.

On the other hand, it was found that some delayed neural networks can exhibit chaotic behavior [11-13]. These kinds of chaotic neural networks have been utilized to solve optimization problems [14]. Since the drive-response concept for considering synchronization of coupled chaotic systems was proposed in 1990 [15], the synchronization of chaotic systems has attracted considerable attention due to its benefits of 
having chaos synchronization in some engineering applications such as secure communication, chemical reactions, information processing and harmonic oscillation generation [16]. Therefore, some chaotic neural networks with delays could be treated as models when we study the synchronization.

Recently, some works dealing with synchronization phenomena in delayed neural networks have also appeared, for example, see [17-29] and references therein. In [17-20], the coupled connected neural networks with delays were considered, several sufficient conditions for synchronization of such neural networks were obtained by Lyapunov stability theory and the linear matrix inequality (LMI) technique. In [21-29], the authors investigated the synchronization problem of some chaotic neural networks with delays. Using the drive-response concept, the control laws were derived to achieve the synchronization of two identical chaotic neural networks.

It is worth pointing out that, the reported works in [17-29] focused on synchronizing of two identical chaotic neural networks with different initial conditions. In practice, the chaotic systems are inevitably subject to some environmental changes, which may render their parameters to be variant. Furthermore, from the point of view of engineering, it is very difficult to keep the two chaotic systems to be identical all the time. Therefore, it is important to study the synchronization problem of nonidentical chaotic neural networks. Obviously, when the considered drive and response neural networks are distinct and with time delay, it becomes more complex and challenging. On the study for synchronization problem of two nonidentical chaotic systems, one usually adopts adaptive control approach to establish synchronization conditions, for example, see [30-32], and references therein. Recently, the integral sliding mode control approach is also employed to investigate synchronization of nonidentical chaotic delayed neural networks [33-38]. In [33], an integral sliding mode control approach is proposed to address synchronization for two nonidentical chaotic neural networks with constant delay. Based on the drive-response concept and Lyapunov stability theory, both delay-independent and delay-dependent conditions in LMIs are derived under which the resulting error system is globally asymptotically stable in the specified switching surface, and a sliding mode controller is synthesized to guarantee the reachability of the specified sliding surface. In [34], the authors investigated synchronization for two chaotic neural networks with discrete and distributed constant delays. By using Lyapunov functional method and LMI technique, a delay-dependent condition was obtained to ensure that the drive system synchronizes with the identical response system. When the parameters and activation functions of two chaotic neural networks mismatched, the synchronization criterion is also derived by sliding mode control approach. In [35], the projective synchronization for two nonidentical chaotic neural networks with constant delay was investigated, a delay-dependent sufficient condition was derived by sliding mode control approach, LMI technique and Lyapunov stability theory. However, to the best of the authors' knowledge, there are no results on the problem of synchronization for chaotic neural networks with leakage delay. As pointed out in [39], neural networks with leakage delay is a class of important neural networks; time delay in the leakage term also has great impact on the dynamics of neural networks because time delay in the stabilizing negative feedback term has a tendency to destabilize a system [39-43]. Therefore, it is necessary to further investigate the synchronization problem for two chaotic neural networks with leakage delay. 
Motivated by the above discussions, the objective of this paper is to present a systematic design procedure for synchronization of two nonidentical chaotic neural networks with discrete and distributed time-varying delays as well as leakage delay. By constructing a proper sliding surface and Lyapunov-Krasovskii functional, and employing a combination of the free-weighting matrix method, Newton-Leibniz formulation and inequality technique, a sliding mode controller is designed to achieve the asymptotical synchronization of the addressed nonidentical neural networks. Moreover, a sliding mode control law is also synthesized to guarantee the reachability of the specified sliding surface. The provided conditions are expressed in terms of LMI, and are dependent on the discrete and distributed time delays as well as leakage delay. Differing from the results in [33-35], the main contributions of this study are to investigate the effect of the leakage delay on the synchronization of two nonidentical chaotic neural networks with discrete and distributed time-varying delays as well as leakage delay and to propose an integral sliding mode control approach to solving it.

\section{Problem formulation and preliminaries}

In this paper, we consider the following neural network model

$$
\begin{aligned}
\dot{y}(t)= & -D_{1} y(t-\delta)+A_{1} f(\gamma(t))+B_{1} f(y(t-\tau(t))) \\
& +C_{1} \int_{t-\sigma(t)}^{t} f(\gamma(s)) d s+I_{1}(t), \quad t \geq 0,
\end{aligned}
$$

where $y(t)=\left(y_{1}(t), y_{2}(t), \ldots, y_{n}(t)\right)^{T} \in R^{n}$ is the state vector of the network at time $t, n$ corresponds to the number of neurons; $D_{1} \in R^{n \times n}$ is a positive diagonal matrix, $A_{1}$, $B_{1}, C_{1} \in R^{n \times n}$ are, respectively, the connection weight matrix, the discretely delayed connection weight matrix and distributively delayed connection weight matrix; $f(y(t))=$ $\left(f_{1}\left(y_{1}(t)\right), f_{2}\left(y_{2}(t)\right), \ldots, f_{n}\left(y_{n}(t)\right)\right)^{T} \in R^{n}$ denotes the neuron activation at time $t ; I_{1}(t) \in R^{n}$ is an external input vector; $\delta \geq 0, \tau(t) \geq 0$ and $\sigma(t) \geq 0$ denote the leakage delay, the discrete time-varying delay and the distributed time-varying delay, respectively, and satisfy $0 \leq \tau(t) \leq \tau, 0 \leq \sigma(t) \leq \sigma$, where $\delta, \tau$ and $\sigma$ are constants. It is assumed that the measured output of system (1) is dependent on the state and the delayed states with the following form:

$$
w(t)=K_{1} \gamma(t)+K_{2} \gamma(t-\delta)+K_{3} \gamma(t-\tau(t))+K_{4} y(t-\sigma(t)),
$$

where $w(t) \in R^{m}, K_{i} \in R^{m \times n}(i=1,2,3,4)$ are known constant matrices.

The initial condition associated with model (1) is given by

$$
\gamma(s)=\phi(s), \quad s \in[-\rho, 0],
$$

where $\varphi(s)$ is bounded and continuously differential on $[-\rho, 0], \rho=\max \{\delta, \tau, \sigma\}$.

We consider the system (1) as the drive system. The response system is as follows:

$$
\begin{aligned}
\dot{z}(t)= & -D_{2} z(t-\delta)+A_{2} g(z(t))+B_{2} g(z(t-\tau(t))) \\
& +C_{2} \int_{t-\sigma(t)}^{t} g(z(s)) d s+I_{2}(t)+u(t), \quad t \geq 0,
\end{aligned}
$$

with initial condition $z(s)=\phi(s), s \in[-\rho, 0]$, where $\phi(s)$ is bounded and continuously differential on $[-\rho, 0], u(t)$ is the appropriate control input that will be designed in order to obtain a certain control objective. 
Let $x(t)=y(t)-z(t)$ be the error state, then the error system can be obtained from (1) and (3) as follows:

$$
\begin{aligned}
\dot{x}(t)= & -D_{1} x(t-\delta)+A_{1} h(x(t))+B_{1} h(x(t-\tau(t)))+C_{1} \int_{t-\sigma(t)}^{t} h(x(s)) d s \\
& +\left(D_{2}-D_{1}\right) z(t-\delta)-A_{2} g(z(t))-B_{2} g(z(t-\tau(t))) \\
& -C_{2} \int_{t-\sigma(t)}^{t} g(z(s)) d s+A_{1} f(z(t))+B_{1} f(z(t-\tau(t))) \\
& +C_{1} \int_{t-\sigma(t)}^{t} f(z(s)) d s-u(t)+I_{1}(t)-I_{2}(t),
\end{aligned}
$$

where $h(x(t))=f(y(t)-f(z(t))$, and $x(s)=\varphi(s)-\phi(s), s \in[-\rho, 0]$.

Definition 1 The drive system (1) and the response system (3) is said to be globally asymptotically synchronized, if system (4) is globally asymptotically stable.

The aim of the paper is to design a controller $u(t)$ to let the response system (3) synchronize with the drive system (1).

Since dynamic behavior of error system (4) relies on both error state $x(t)$ and chaotic state $z(t)$ of response system (3), complete synchronization between two nonidentical chaotic neural networks (1) and (3) cannot be achieved only by utilizing output feedback control. To overcome the difficulty, an integral sliding mode control approach will be proposed to investigate the synchronization problem of two nonidentical chaotic neural networks (1) and (3). In other words, an integral sliding mode controller is designed such that the sliding motion is globally asymptotically stable, and the state trajectory of the error system (4) is globally driven onto the specified sliding surface and maintained there for all subsequent time.

To utilize the information of the measured output $w(t)$, a suitable sliding surface is constructed as

$$
\begin{aligned}
S(t)= & x(t)+\int_{0}^{t}\left[D_{1} x(\xi-\delta)-A_{1} h(x(\xi))-B_{1} h(x(\xi-\tau(\xi)))\right. \\
& -C_{1} \int_{\xi-\sigma(\xi)}^{\xi} h(x(s)) d s+K\left(w(\xi)-K_{1} z(\xi)-K_{2} z(\xi-\delta)\right. \\
& \left.\left.-K_{3} z(\xi-\tau(\xi))-K_{4} z(\xi-\sigma(\xi))\right)\right] d \xi,
\end{aligned}
$$

where $K \in R^{n \times m}$ is a gain matrix to be determined.

It follows from (2), (4) and (5) that

$$
\begin{aligned}
S(t)= & x(0)+\int_{0}^{t}\left[\left(D_{2}-D_{1}\right) z(\xi-\delta)-A_{2} g(z(\xi))-B_{2} g(z(\xi-\tau(\xi)))\right. \\
& -C_{2} \int_{\xi-\sigma(\xi)}^{\xi} g(z(s)) d s+A_{1} f(z(\xi))+B_{1} f(z(\xi-\tau(\xi))) \\
& +C_{1} \int_{\xi-\sigma(\xi)}^{\xi} f(z(s)) d s-u(\xi)+I_{1}(\xi)-I_{2}(\xi)+K K_{1} x(\xi) \\
& \left.+K K_{2} x(\xi-\delta)+K K_{3} x(\xi-\tau(\xi))+K K_{4} x(\xi-\sigma(\xi))\right] d \xi
\end{aligned}
$$

According to the sliding mode control theory [44], it is true that $S(t)=0$ and $\dot{S}(t)=0$ as the state trajectories of the error system (4) enter into the sliding mode. It 
thus follows from (6) and $\dot{S}(t)=0$ that an equivalent control law can be designed as

$$
\begin{aligned}
u(t)= & \left(D_{2}-D_{1}\right) z(t-\delta)-A_{2} g(z(t))-B_{2} g(z(t-\tau(t))) \\
& -C_{2} \int_{t-\sigma(t)}^{t} g(z(s)) d s+A_{1} f(z(t))+B_{1} f(z(t-\tau(t))) \\
& +C_{1} \int_{t-\sigma(t)}^{t} f(z(s)) d s+I_{1}(t)-I_{2}(t) \\
& +K K_{1} x(t)+K K_{2} x(t-\delta)+K K_{3} x(t-\tau(t))+K K_{4} x(t-\sigma(t)) .
\end{aligned}
$$

Substituting (7) into (4), the sliding mode dynamics can be obtained and described by

$$
\begin{aligned}
\dot{x}(t)= & -K K_{1} x(t)-\left(D_{1}+K K_{2}\right) x(t-\delta)-K K_{3} x(t-\tau(t))-K K_{4} x(t-\sigma(t)) \\
& +A_{1} h(x(t))+B_{1} h(x(t-\tau(t)))+C_{1} \int_{t-\sigma(t)}^{t} h(x(s)) d s .
\end{aligned}
$$

Throughout this paper, we make the following assumption:

(H). For any $j \in\{1,2, \ldots, n\}$, there exist constants $F_{j}^{-}, F_{j}^{+}, G_{j}^{-}$and $G_{j}^{+}$such that

$$
F_{j}^{-} \leq \frac{f_{j}\left(\alpha_{1}\right)-f_{j}\left(\alpha_{2}\right)}{\alpha_{1}-\alpha_{2}} \leq F_{j}^{+}, \quad G_{j}^{-} \leq \frac{g_{j}\left(\alpha_{1}\right)-g_{j}\left(\alpha_{2}\right)}{\alpha_{1}-\alpha_{2}} \leq G_{j}^{+}
$$

for all $\alpha_{1} \neq \alpha_{2}$.

To prove our result, the following lemma that can be found in [41] is necessary.

Lemma 1 For any constant matrix $W \in R^{m \times m}, W>0$, scalar $0<h(t)<h$, vector function $\omega:[0, h] \rightarrow R^{m}$ such that the integrations concerned are well defined, then

$$
\left(\int_{0}^{h(t)} \omega(s) d s\right)^{T} W\left(\int_{0}^{h(t)} \omega(s) d s\right) \leq h(t) \int_{0}^{h(t)} \omega^{T}(s) W \omega(s) d s .
$$

\section{Main results}

For presentation convenience, in the following, we denote

$$
\begin{gathered}
F_{1}=\operatorname{diag}\left(F_{1}^{-}, F_{2}^{-}, \ldots, F_{n}^{-}\right), \quad F_{2}=\operatorname{diag}\left(F_{1}^{+}, F_{2}^{+}, \ldots, F_{n}^{+}\right), \\
F_{3}=\operatorname{diag}\left(F_{1}^{-} F_{1}^{+}, F_{2}^{-} F_{2}^{+}, \ldots, F_{n}^{-} F_{n}^{+}\right), \\
F_{4}=\operatorname{diag}\left(\frac{F_{1}^{-}+F_{1}^{+}}{2}, \frac{F_{2}^{-}+F_{2}^{+}}{2}, \ldots, \frac{F_{n}^{-}+F_{n}^{+}}{2}\right) .
\end{gathered}
$$

Theorem 1 Assume that the condition $(\mathbf{H})$ holds and the measured output of drive neural network (1) is condition (2). If there exist five symmetric positive definite matrices $P_{i}(i=1,2,3,4,5)$, four positive diagonal matrices $R_{i}(i=1,2,3,4)$, and ten matrices $M, N, L, Y, X_{i j}(i, j=1,2,3, i \leq j)$ such that the following two LMIs hold:

$$
X=\left[\begin{array}{lll}
X_{11} & X_{12} & X_{13} \\
X_{12}^{T} & X_{22} & X_{23} \\
X_{13}^{T} & X_{23}^{T} & X_{33}
\end{array}\right]>0,
$$




$$
\Omega=\left[\begin{array}{cccccccccc}
\Omega_{11} & \Omega_{12} & \Omega_{13} & \Omega_{14} & \Omega_{15} & \Omega_{16} & \Omega_{17} & \Omega_{18} & \Omega_{19} & \Omega_{1,10} \\
* & -P_{3} & 0 & \Omega_{24} & \Omega_{25} & \Omega_{26} & \Omega_{27} & \Omega_{28} & \Omega_{29} & 0 \\
* & * & \Omega_{33} & \Omega_{34} & -Y K_{3} & -Y K_{4} & \Omega_{37} & P_{1} B_{1} & P_{1} C_{1} & 0 \\
* & * & * & -P_{2} & 0 & 0 & 0 & 0 & 0 & 0 \\
* & * & * & * & \Omega_{55} & 0 & 0 & F_{4} R_{4} & 0 & 0 \\
* & * & * & * & * & \Omega_{66} & 0 & 0 & 0 & \Omega_{6,10} \\
* & * & * & * & * & * & \Omega_{77} & 0 & 0 & 0 \\
* & * & * & * & * & * & * & -R_{4} & 0 & 0 \\
* & * & * & * & * & * & * & * & -P_{4} & 0 \\
* & * & * & * & * & * & * & * & * & \Omega_{10,10}
\end{array}\right]<0,
$$

in $\quad \Omega_{11}=-P_{1} D_{1}-D_{1} P_{1}-Y K_{1}-K_{1}^{T} Y^{T}+P_{2}+\delta^{2} P_{3}+\tau X_{11}+X_{13}+X_{13}^{T}-F_{3} R_{3}+M+M^{T}$, $\Omega_{13}=-F_{1} R_{1}+F_{2} R_{2}-K_{1}^{T} Y^{T}, \quad \Omega_{13}=-F_{1} R_{1}+F_{2} R_{2}-K_{1}^{T} Y^{T}, \quad \Omega_{14} \quad=\quad-Y K_{2}$, $\Omega_{15}=-Y K_{3}+\tau X_{12}-X_{13}+X_{23}^{T}, \Omega_{16}=-Y K_{4}-M^{T}+N, \Omega_{17}=P_{1} A_{1}+F_{4} R_{3}, \Omega_{18}=$ $P_{1} B_{1}, \Omega_{19}=P_{1} C_{1}, \Omega_{1,10}=L-M^{T}, \Omega_{24}=D_{1} Y K_{2}, \Omega_{25}=D_{1} Y K_{3}, \Omega_{26}=D_{1} Y K_{4}, \Omega_{27}=$ $-D_{1} P_{1} A_{1}, \Omega_{28}=D_{1} P_{1} B_{1}, \Omega_{29}=D_{1} P_{1} C_{1}, \Omega_{33}=\tau X_{33}+\sigma^{2} P_{5}-2 P_{1}, \Omega_{34}=-P_{1} D_{1}-Y$ $K_{2}, \Omega_{37}=R_{1}-R_{2}+P_{1} A_{1}, \Omega_{55}=\tau X_{22}-X_{23}-X_{23}^{T}-F_{3} R_{4}, \Omega_{66}=-N-N^{T}, \Omega_{6,10}=-L$ - $N^{T}, \Omega_{77}=\sigma^{2} P_{4}-R_{3}, \Omega_{10,10}=-P_{5}-L-L^{T}$, then the response neural network (3) can globally asymptotically synchronize the drive neural network (1), and the gain matrix $K$ can be designed as

$$
K=P_{1}^{-1} Y \text {. }
$$

Proof 1 Let $R_{i}=\operatorname{diag}\left(r_{1}^{(i)}, r_{2}^{(i)}, \ldots, r_{n}^{(i)}\right)(i=1,2), v(\xi, s)=\left(x^{T}(\xi), x^{T}(\xi-\tau(\xi)), \dot{x}^{T}(s)\right)^{T}$, and consider the following Lyapunov-Krasovskii functional as

$$
V(t)=V_{1}(t)+V_{2}(t)+V_{3}(t)+V_{4}(t)+V_{5}(t)+V_{6}(t)+V_{7}(t)
$$

where

$$
\begin{aligned}
& V_{1}(t)=\left(x(t)-D_{1} \int_{t-\delta}^{t} x(s) d s\right)^{T} P_{1}\left(x(t)-D_{1} \int_{t-\delta}^{t} x(s) d s\right) \\
& V_{2}(t)=2 \sum_{i=1}^{n} r_{i}^{(1)} \int_{0}^{x_{i}(t)}\left(h_{i}(s)-F_{i}^{-} s\right) d s+2 \sum_{i=1}^{n} r_{i}^{(2)} \int_{0}^{x_{i}(t)}\left(F_{i}^{+} s-h_{i}(s)\right) d s, \\
& V_{3}(t)=\int_{t-\delta}^{t} x^{T}(s) P_{2} x(s) d s+\delta \int_{-\delta}^{0} \int_{t+\xi}^{t} x^{T}(s) P_{3} x(s) d s d \xi \\
& V_{4}(t)=\int_{-\tau}^{0} \int_{t+\xi}^{t} \dot{x}^{T}(s) X_{33} \dot{x}(s) d s d \xi \\
& V_{5}(t)=\sigma \int_{-\sigma}^{0} \int_{t+\xi}^{t} h^{T}(x(s)) P_{4} h(x(s)) d s d \xi \\
& V_{6}(t)=\sigma \int_{-\sigma}^{0} \int_{t+\xi}^{t} \dot{x}^{T}(s) P_{5} \dot{x}(s) d s d \xi \\
& V_{7}(t)=\int_{0}^{t} \int_{\xi-\tau(\xi)}^{\xi} v^{T}(\xi, s) X v(\xi, s) d s d \xi .
\end{aligned}
$$


Calculating the time derivative of $V_{1}(t)$ along the trajectories of model (8), we obtain

$$
\begin{aligned}
\dot{V}_{1}(t)= & 2\left(x(t)-D_{1} \int_{t-\delta}^{t} x(s) d s\right)^{T} P_{1}\left(-\left(D_{1}+K K_{1}\right) x(t)-K K_{2} x(t-\delta)\right. \\
& -K K_{3} x(t-\tau(t))-K K_{4} x(t-\sigma(t))+A_{1} h(x(t)) \\
& \left.+B_{1} h(x(t-\tau(t)))+C_{1} \int_{t-\sigma(t)}^{t} h(x(s)) d s\right) \\
= & x^{T}(t)\left(-2 P_{1} D_{1}-2 P_{1} K K_{1}\right) x(t) \\
& +2 x^{T}(t)\left(D_{1} P_{1} D_{1}+K_{1}^{T} K^{T} P_{1} D_{1}\right) \int_{t-\delta}^{t} x(s) d s \\
& -2 x^{T}(t) P_{1} K K_{2} x(t-\delta)-2 x^{T}(t) P_{1} K K_{3} x(t-\tau(t)) \\
& -2 x^{T}(t) P_{1} K K_{4} x(t-\sigma(t))+2 x^{T}(t) P_{1} A_{1} h(x(t)) \\
& +2 x^{T}(t) P_{1} B_{1} h(x(t-\tau(t)))+2 x^{T}(t) P_{1} C_{1} \int_{t-\sigma(t)}^{t} h(x(s)) d s \\
& +2\left(\int_{t-\delta}^{t} x(s) d s\right)^{T} D_{1} P_{1} K K_{2} x(t-\delta) \\
& +2\left(\int_{t-\delta}^{t} x(s) d s\right)^{T} D_{1} P_{1} K K_{3} x(t-\tau(t)) \\
& +2\left(\int_{t-\delta}^{t} x(s) d s\right)^{T} D_{1} P_{1} K K_{4} x(t-\sigma(t)) \\
& -2\left(\int_{t-\delta}^{t} x(s) d s\right)^{T} D_{1} P_{1} A_{1} h(x(t)) \\
& -2\left(\int_{t-\delta}^{t} x(s) d s\right)^{T} D_{1} P_{1} B_{1} h(x(t-\tau(t))) \\
& -2\left(\int_{t-\delta}^{t} x(s) d s\right)^{T} D_{1} P_{1} C_{1} \int_{t-\sigma(t)}^{t} h(x(s)) d s .
\end{aligned}
$$

Calculating the time derivatives of $V_{i}(t)(i=2,3,4,5,6,7)$, we have

$$
\begin{aligned}
\dot{V}_{2}(t)= & 2 \dot{x}^{T}(t) R_{1}\left(h(x(t))-F_{1} x(t)\right)+2 \dot{x}^{T}(t) R_{2}\left(F_{2} x(t)-h(x(t))\right) \\
= & 2 x^{T}(t)\left(-F_{1} R_{1}+F_{2} R_{2}\right) \dot{x}(t)+2 \dot{x}^{T}(t)\left(R_{1}-R_{2}\right) h(x(t)), \\
\dot{V}_{3}(t)= & x^{T}(t)\left(P_{2}+\delta^{2} P_{3}\right) x(t)-x^{T}(t-\delta) P_{2} x(t-\delta)-\delta \int_{t-\delta}^{t} x^{T}(s) P_{3} x(s) d s \\
\leq & x^{T}(t)\left(P_{2}+\delta^{2} P_{3}\right) x(t)-x^{T}(t-\delta) P_{2} x(t-\delta) \\
& -\left(\int_{t-\delta}^{t} x(s) d s\right)^{T} P_{3}\left(\int_{t-\delta}^{t} x(s) d s\right) \\
\dot{V}_{4}(t)= & \tau \dot{x}^{T}(t) X_{33} \dot{x}(t)-\int_{t-\tau}^{t} \dot{x}^{T}(s) X_{33} \dot{x}(s) d s, \\
\dot{V}_{5}(t)= & \sigma^{2} h^{T}(x(t)) P_{4} h(x(t))-\sigma \int_{t-\sigma}^{t} h^{T}(x(s)) P_{4} h(x(s)) d s \\
\leq & \sigma^{2} h^{T}(x(t)) P_{4} h(x(t))-\sigma(t) \int_{t-\sigma(t)}^{t} h^{T}(x(s)) P_{4} h(x(s)) d s \\
\leq & \sigma^{2} h^{T}(x(t)) P_{4} h(x(t)) \\
& -\left(\int_{t-\sigma(t)}^{t} h(x(s)) d s\right)^{T} P_{4}\left(\int_{t-\sigma(t)}^{t} h(x(s)) d s\right),
\end{aligned}
$$




$$
\begin{aligned}
\dot{V}_{6}(t)= & \sigma^{2} \dot{x}^{T}(t) P_{5} \dot{x}(t)-\sigma \int_{t-\sigma}^{t} \dot{x}^{T}(s) P_{5} \dot{x}(s) d s \\
\leq & \sigma^{2} \dot{x}^{T}(t) P_{5} \dot{x}(t)-\sigma(t) \int_{t-\sigma(t)}^{t} \dot{x}^{T}(s) P_{5} \dot{x}(s) d s \\
\leq & \sigma^{2} \dot{x}^{T}(t) P_{5} \dot{x}(t)-\left(\int_{t-\sigma(t)}^{t} \dot{x}(s) d s\right)^{T} P_{5}\left(\int_{t-\sigma(t)}^{t} \dot{x}(s) d s\right), \\
\dot{V}_{7}(t)= & \int_{t-\tau(t)}^{t} v^{T}(t, s) X v(t, s) d s \\
= & \left.\tau(t)\left(\begin{array}{c}
x(t) \\
x(t-\tau(t))
\end{array}\right)\right)^{T}\left(\begin{array}{c}
X_{11} X_{12} \\
X_{12}^{T} X_{22}
\end{array}\right)\left(\begin{array}{c}
x(t) \\
x(t-\tau(t))
\end{array}\right)+2 x^{T}(t) X_{13} x(t) \\
& -2 x^{T}(t) X_{13} x(t-\tau(t))+2 x^{T}(t-\tau(t)) X_{23} x(t) \\
& -2 x^{T}(t-\tau(t)) X_{23} x(t-\tau(t))+\int_{t-\tau(t)}^{t} \dot{x}^{T}(s) X_{33} \dot{x}(s) d s \\
\leq & x^{T}(t)\left(\tau X_{11}+2 X_{13}\right) x(t)+2 x^{T}(t)\left(\tau X_{12}-X_{13}+X_{23}^{T}\right) x(t-\tau(t)) \\
& +x^{T}(t-\tau(t))\left(\tau X_{22}-2 X_{23}\right) x(t-\tau(t))+\int_{t-\tau}^{t} \dot{x}^{T}(s) X_{33} \dot{x}(s) d s .
\end{aligned}
$$

In deriving inequalities (22), (24) and (25), we have made use of $0 \leq \sigma(t) \leq \sigma, 0 \leq \tau(t)$ $\leq \tau$ and Lemma 1. It follows from inequalities (20)-(26) that

$$
\begin{aligned}
\dot{V}(t) \leq & x^{T}(t)\left(-2 P_{1} D_{1}-2 P_{1} K K_{1}+P_{2}+\delta^{2} P_{3}+\tau X_{11}+2 X_{13}\right) x(t) \\
& +2 x^{T}(t)\left(D_{1} P_{1} D_{1}+K_{1}^{T} K^{T} P_{1} D_{1}\right) \int_{t-\delta}^{t} x(s) d s \\
& +2 x^{T}(t)\left(-F_{1} R_{1}+F_{2} R_{2}\right) \dot{x}(t)-2 x^{T}(t) P_{1} K K_{2} x(t-\delta) \\
& +2 x^{T}(t)\left(-P_{1} K K_{3}+\tau X_{12}-X_{13}+X_{23}^{T}\right) x(t-\tau(t)) \\
& -2 x^{T}(t) P_{1} K K_{4} x(t-\sigma(t))+2 x^{T}(t) P_{1} A_{1} h(x(t)) \\
& +2 x^{T}(t) P_{1} B_{1} h(x(t-\tau(t)))+2 x^{T}(t) P_{1} C_{1} \int_{t-\sigma(t)}^{t} h(x(s)) d s \\
& -\left(\int_{t-\delta}^{t} x(s) d s\right)^{T} P_{3}\left(\int_{t-\delta}^{t} x(s) d s\right) \\
& +2\left(\int_{t-\delta}^{t} x(s) d s\right)^{T} D_{1} P_{1} K K_{2} x(t-\delta) \\
& +2\left(\int_{t-\delta}^{t} x(s) d s\right)^{T} D_{1} P_{1} K K_{3} x(t-\tau(t)) \\
& +2\left(\int_{t-\delta}^{t} x(s) d s\right)^{T} D_{1} P_{1} K K_{4} x(t-\sigma(t)) \\
& -2\left(\int_{t-\delta}^{t} x(s) d s\right)^{T} D_{1} P_{1} A_{1} h(x(t)) \\
& -2\left(\int_{t-\delta}^{t} x(s) d s\right)^{T} D_{1} P_{1} B_{1} h(x(t-\tau(t))) \\
& -2\left(\int_{t-\delta}^{t} x(s) d s\right)^{T} D_{1} P_{1} C_{1} \int_{t-\sigma(t)}^{t} h(x(s)) d s \\
& +\dot{x}^{T}(t)\left(\tau X_{33}+\sigma^{2} P_{5}\right) \dot{x}(t)+2 \dot{x}^{T}(t)\left(R_{1}-R_{2}\right) h(x(t)) \\
& -x^{T}(t-\delta) P_{2} x(t-\delta)+x^{T}(t-\tau(t))\left(\tau X_{22}-2 X_{23}\right) x(t-\tau(t)) \\
& +\sigma^{2} h^{T}(x(t)) P_{4} h(x(t))-\left(\int_{t-\sigma(t)}^{t} h(x(s)) d s\right)^{T} P_{4}\left(\int_{t-\sigma(t)}^{t} h(x(s)) d s\right) \\
& -\left(\int_{t-\sigma(t)}^{t} \dot{x}(s) d s\right)^{T} P_{5}\left(\int_{t-\sigma(t)}^{t} \dot{x}(s) d s\right) \\
= & \alpha^{T}(t) \Pi \alpha(t),
\end{aligned}
$$


where

$$
\begin{aligned}
& \alpha(t)=\left(x^{T}(t), \int_{t-\delta}^{t} x(s) d s, \dot{x}^{T}(t), x^{T}(t-\delta), x^{T}(t-\tau(t)), x^{T}(t-\sigma(t)),\right. \\
& \left.h^{T}(x(t)), h^{T}(x(t-\tau(t))), \int_{t-\sigma(t)}^{t} h^{T}(x(s)) d s, \int_{t-\sigma(t)}^{t} \dot{x}^{T}(s) d s\right)^{T}, \\
& \Pi=\left[\begin{array}{cccccccccc}
\Pi_{11} & \Pi_{12} & \Pi_{13} & \Pi_{14} & \Pi_{15} & \Pi_{16} & P_{1} A_{1} & P_{1} B_{1} & P_{1} C_{1} & 0 \\
* & -P_{3} & 0 & \Pi_{24} & \Pi_{25} & \Pi_{26} & \Pi_{27} & \Pi_{28} & \Pi_{29} & 0 \\
* & * & \Pi_{33} & 0 & 0 & 0 & R_{1}-R_{2} & 0 & 0 & 0 \\
* & * & * & -P_{2} & 0 & 0 & 0 & 0 & 0 & 0 \\
* & * & * & * & \Pi_{55} & 0 & 0 & 0 & 0 & 0 \\
* & * & * & * & * & 0 & 0 & 0 & 0 & 0 \\
* & * & * & * & * & * & \sigma^{2} P_{4} & 0 & 0 & 0 \\
* & * & * & * & * & * & * & 0 & 0 & 0 \\
* & * & * & * & * & * & * & * & -P_{4} & 0 \\
* & * & * & * & * & * & * & * & * & -P_{5}
\end{array}\right]
\end{aligned}
$$

with

$$
\Pi_{11}=-P_{1} D_{1}-D_{1} P_{1}-P_{1} K K_{1}-K_{1}^{T} K^{T} P_{1}+P_{2}+\delta^{2} P_{3}+\tau X_{11}+X_{13}+X_{13}^{T},
$$

$\Pi_{12}=D_{1} P_{1} D_{1}+K_{1}^{T} K^{T} P_{1} D_{1}, \quad \Pi_{13}=-F_{1} R_{1}+F_{2} R_{2}, \quad \Pi_{14}=-P_{1} K K_{2}$,

$\Pi_{15}=-P_{1} K K_{3}+\tau X_{12}-X_{13}+X_{23}^{T}, \Pi_{16}=-P_{1} K K_{4}, \Pi_{24}=D_{1} P_{1} K K_{2}, \Pi_{25}=D_{1} P_{1} K K_{3}, \Pi_{26}$ $=D_{1} P_{1} K K_{4}, \Pi_{27}=-D_{1} P_{1} A_{1}, \Pi_{28}=-D_{1} P_{1} B_{1}, \Pi_{29}=-D_{1} P_{1} C_{1}, \Pi_{33}=\tau X_{33}+\sigma^{2} P_{5}$, $\Pi_{55}=\tau X_{22}-X_{23}-X_{23}^{T}$.

In addition, for any $n \times n$ diagonal matrices $R_{3}>0$ and $R_{4}>0$, we can get from assumption $(\mathbf{H})$ that [45]

$$
\begin{aligned}
& {\left[\begin{array}{c}
x(t) \\
h(x(t))
\end{array}\right]^{T}\left[\begin{array}{cc}
F_{3} R_{3} & -F_{4} R_{3} \\
-F_{4} R_{3} & R_{3}
\end{array}\right]\left[\begin{array}{c}
x(t) \\
h(x(t))
\end{array}\right] \leq 0} \\
& {\left[\begin{array}{c}
x(t-\tau(t)) \\
h(x(t-\tau(t)))
\end{array}\right]^{T}\left[\begin{array}{cc}
F_{3} R_{4} & -F_{4} R_{4} \\
-F_{4} R_{4} & R_{4}
\end{array}\right]\left[\begin{array}{c}
x(t-\tau(t)) \\
h(x(t-\tau(t)))
\end{array}\right] \leq 0 .}
\end{aligned}
$$

From Newton-Leibniz formulation $x(t)-x(t-\sigma(t))-\int_{t-\sigma(t)}^{t} \dot{x}(s) d s=0$, we have

$$
\begin{aligned}
0= & 2\left(x(t)-x(t-\sigma(t))-\int_{t-\sigma(t)}^{t} \dot{x}(s) d s\right)^{T} \\
& \times\left(M x(t)+N x(t-\sigma(t))+L \int_{t-\sigma(t)}^{t} \dot{x}(s) d s\right) .
\end{aligned}
$$

Noting this fact

$$
\begin{aligned}
0= & 2 \dot{x}^{T}(t) P_{1}\left(-\dot{x}(t)-K K_{1} x(t)-\left(D_{1}+K K_{2}\right) x(t-\delta)\right. \\
& -K K_{3} x(t-\tau(t))-K K_{4} x(t-\sigma(t))+A_{1} h(x(t)) \\
& \left.+B_{1} h(x(t-\tau(t)))+C_{1} \int_{t-\sigma(t)}^{t} h(x(s)) d s\right) .
\end{aligned}
$$


It follows from (27)-(31) that

$$
\begin{aligned}
\dot{V}(t) \leq & \alpha^{T}(t) \Pi \alpha(t)-\left[\begin{array}{c}
x(t) \\
h(x(t))
\end{array}\right]^{T}\left[\begin{array}{cc}
F_{3} R_{3} & -F_{4} R_{3} \\
-F_{4} R_{3} & R_{3}
\end{array}\right]\left[\begin{array}{c}
x(t) \\
h(x(t))
\end{array}\right] \\
& -\left[\begin{array}{c}
x(t-\tau(t)) \\
h(x(t-\tau(t)))
\end{array}\right]^{T}\left[\begin{array}{cc}
F_{3} R_{4} & -F_{4} R_{4} \\
-F_{4} R_{4} & R_{4}
\end{array}\right]\left[\begin{array}{c}
x(t-\tau(t)) \\
h(x(t-\tau(t)))
\end{array}\right] \\
& +2\left(x(t)-x(t-\sigma(t))-\int_{t-\sigma(t)}^{t} \dot{x}(s) d s\right)^{T} \\
& \times\left(M x(t)+N x(t-\sigma(t))+L \int_{t-\sigma(t)}^{t} \dot{x}(s) d s\right) \\
& +2 \dot{x}^{T}(t) P_{1}\left(-\dot{x}(t)-K K_{1} x(t)-\left(D_{1}+K K_{2}\right) x(t-\delta)\right. \\
& -K K_{3} x(t-\tau(t))-K K_{4} x(t-\sigma(t))+A_{1} h(x(t)) \\
& \left.+B_{1} h(x(t-\tau(t)))+C_{1} \int_{t-\sigma(t)}^{t} h(x(s)) d s\right) \\
= & \alpha^{T}(t) \Gamma \alpha(t),
\end{aligned}
$$

where

$$
\Gamma=\left[\begin{array}{cccccccccc}
\Gamma_{11} & \Pi_{12} & \Gamma_{13} & \Pi_{14} & \Pi_{15} & \Gamma_{16} & \Gamma_{17} & P_{1} B_{1} & P_{1} C_{1} & \Gamma_{1,10} \\
* & -P_{3} & 0 & \Pi_{24} & \Pi_{25} & \Pi_{26} & \Pi_{27} & \Pi_{28} & \Pi_{29} & 0 \\
* & * & \Gamma_{33} & \Gamma_{34} & -P_{1} K K_{3} & -P_{1} K K_{4} & \Gamma_{37} & P_{1} B_{1} & P_{1} C_{1} & 0 \\
* & * & * & -P_{2} & 0 & 0 & 0 & 0 & 0 & 0 \\
* & * & * & * & \Gamma_{55} & 0 & 0 & F_{4} R_{4} & 0 & 0 \\
* & * & * & * & * & \Gamma_{66} & 0 & 0 & 0 & \Gamma_{6,10} \\
* & * & * & * & * & * & \Gamma_{77} & 0 & 0 & 0 \\
* & * & * & * & * & * & * & -R_{4} & 0 & 0 \\
* & * & * & * & * & * & * & * & -P_{4} & 0 \\
* & * & * & * & * & * & * & * & * & \Gamma_{10,10}
\end{array}\right]
$$

with $\Gamma_{11}=\Gamma_{11}-F_{3} R_{3}+M+M^{T}, \Gamma_{13}=\Pi_{13}-K_{1}^{T} K^{T} P_{1}, \Gamma_{16}=-P_{1} K K_{4} M^{T}+N, \Gamma_{17}=$ $P_{1} A_{1}+F_{4} R_{3}, \Gamma_{1,10}=L-M^{T}, \Gamma_{33}=\Pi_{33}-2 P_{1}, \Gamma_{34}=-P_{1} D_{1}-P_{1} K K_{2}, \Gamma_{37}=R_{1}-R_{2}$ $+P_{1} A_{1}, \Gamma_{55}=\Pi_{55}-F_{3} R_{4}, \Gamma_{66}=-N-N^{T}, \Gamma_{6,10}=-L-N^{T}, \Gamma_{77}=\sigma^{2} P_{4}-R_{3}, \Gamma_{10,10}=-P 5$ $-L-L^{T}$.

From (10) and (11), we get that $\Gamma=\Omega<0$. There must exist a small scalar $\rho>0$ such that

$$
\Gamma+\operatorname{diag}\{\rho I, 0,0,0,0,0,0,0,0,0\} \leq 0 .
$$

It follows from (32) and (33), we get that

$$
\dot{V}(t) \leq-\rho \alpha^{T}(t) \alpha(t) \leq-\rho x^{T}(t) x(t), \quad t \geq 0,
$$

which implies that the error dynamical system (8) is globally asymptotically stable by the Lyapunov stability theory. Accordingly, the response neural network (3) can globally asymptotically synchronize the drive neural network (1). The proof is completed.

When there is no leakage delay, the drive neural network (1) and the response neural network (3) become, respectively, the following models

$$
\begin{aligned}
\dot{y}(t)= & -D_{1} \gamma(t)+A_{1} f(y(t))+B_{1} f(\gamma(t-\tau(t))) \\
& +C_{1} \int_{t-\sigma(t)}^{t} f(\gamma(s)) d s+I_{1}(t), \quad t \geq 0,
\end{aligned}
$$


and

$$
\begin{aligned}
\dot{z}(t)= & -D_{2} z(t)+A_{2} g(z(t))+B_{2} g(z(t-\tau(t))) \\
& +C_{2} \int_{t-\sigma(t)}^{t} g(z(s)) d s+I_{2}(t)+u(t), \quad t \geq 0 .
\end{aligned}
$$

It is assumed that the measured output of system (34) is dependent on the state and the delayed states with the following form:

$$
w(t)=K_{1} \gamma(t)+K_{3} \gamma(t-\tau(t))+K_{4} \gamma(t-\sigma(t)),
$$

where $w(t) \in R^{m}, K_{i} \in R^{m \times n}(i=1,3,4)$ are known constant matrices.

From the process of proof in Theorem 1, we can get the following result.

Corollary 1 Assume that the condition $(\mathbf{H})$ holds and the measured output of drive neural network (34) is condition (36). If there exist three symmetric positive definite matrices $P_{i}(i=1,4,5)$, four positive diagonal matrices $R_{i}(i=1,2,3,4)$, and ten matrices $M, N, L, Y, X_{i j}(i, j=1,2,3, i \leq j)$ such that the following two LMIs hold:

$$
\begin{aligned}
X & =\left[\begin{array}{ccc}
X_{11} & X_{12} & X_{13} \\
X_{12}^{T} & X_{22} & X_{23} \\
X_{13}^{T} & X_{23}^{T} & X_{33}
\end{array}\right]>0, \\
\Omega & =\left[\begin{array}{cccccccc}
\Omega_{11} & \Omega_{13} & \Omega_{15} & \Omega_{16} & \Omega_{17} & \Omega_{18} & \Omega_{19} & \Omega_{1,10} \\
* & \Omega_{33} & -Y K_{3} & -Y K_{4} & \Omega_{37} & P_{1} B_{1} & P_{1} C_{1} & 0 \\
* & * & \Omega_{55} & 0 & 0 & F_{4} R_{4} & 0 & 0 \\
* & * & * & \Omega_{66} & 0 & 0 & 0 & \Omega_{6,10} \\
* & * & * & * & \Omega_{77} & 0 & 0 & 0 \\
* & * & * & * & * & -R_{4} & 0 & 0 \\
* & * & * & * & * & * & -P_{4} & 0 \\
* & * & * & * & * & * & * & \Omega_{10,10}
\end{array}\right]<0,
\end{aligned}
$$

in $\quad \Omega_{11}=-P_{1} D_{1}-D_{1} P_{1}-Y K_{1}-K_{1}^{T} Y^{T}+\tau X_{11}+X_{13}+X_{13}^{T}-F_{3} R_{3}+M+M^{T}$, $\Omega_{15}=-Y K_{3}+\tau X_{12}-X_{13}+X_{23}^{T}, \Omega_{15}=-Y K_{3}+\tau X_{12}-X_{13}+X_{23}^{T}, \Omega_{16}=-Y K_{4}-M^{T}$ $+N, \Omega_{17}=P_{1} A_{1}+F_{4} R_{3}, \Omega_{18}=P_{1} B_{1}, \Omega_{19}=P_{1} C_{1}, \Omega_{1,10}=L-M^{T}, \Omega_{33}=\tau X_{33}+\sigma^{2} P_{5}$ $-2 P_{1}, \Omega_{37}=R_{1}-R_{2}+P_{1} A_{1}, \Omega_{55}=\tau X_{22}-X_{23}-X_{23}^{T}-F_{3} R_{4}, \Omega_{66}=-N-N^{T}, \Omega_{6,10}=-$ $L-N^{T}, \Omega_{77}=\sigma^{2} P_{4}-R_{3}, \Omega_{10,10}=-P_{5}-L-L^{T}$, then the response neural network (35) can globally asymptotically synchronize the drive neural network (34), and the gain matrix $K$ can be designed as

$$
K=P_{1}^{-1} Y .
$$

When there is no both leakage delay and distributed time-varying delays, the drive neural network (1) and the response neural network (3) become, respectively, the following models

$$
\dot{\gamma}(t)=-D_{1} \gamma(t)+A_{1} f(\gamma(t))+B_{1} f(\gamma(t-\tau(t)))+I_{1}(t), \quad t \geq 0,
$$

and

$$
\dot{z}(t)=-D_{2} z(t)+A_{2} g(z(t))+B_{2} g(z(t-\tau(t)))+I_{2}(t)+u(t), \quad t \geq 0 .
$$

It is assumed that the measured output of system (46) is dependent on the state and the delayed states with the following form:

$$
w(t)=K_{1} \gamma(t)+K_{3} \gamma(t-\tau(t)),
$$


where $w(t) \in R^{m}, K_{i} \in R^{m \times n}(i=1,3)$ are known constant matrices.

From the process of proof in Theorem 1, we can get the following result.

Corollary 2 Assume that the condition $(\mathbf{H})$ holds and the measured output of drive neural network (40) is condition (42). If there exist a symmetric positive definite matrices $P_{1}$, four positive diagonal matrices $R_{i}(i=1,2,3,4)$, and seven matrices $Y, X_{i j}$ $(i, j=1,2,3, i \leq j)$ such that the following two LMIs hold:

$$
\begin{aligned}
X & =\left[\begin{array}{ccc}
X_{11} & X_{12} & X_{13} \\
X_{12}^{T} & X_{22} & X_{23} \\
X_{13}^{T} & X_{23}^{T} & X_{33}
\end{array}\right]>0, \\
\Omega & =\left[\begin{array}{ccccc}
\Omega_{11} & \Omega_{13} & \Omega_{15} & \Omega_{17} & P_{1} B_{1} \\
* & \Omega_{33} & -Y K_{3} & \Omega_{37} & P_{1} B_{1} \\
* & * & \Omega_{55} & 0 & F_{4} R_{4} \\
* & * & * & -R_{3} & 0 \\
* & * & * & * & -R_{4}
\end{array}\right]<0,
\end{aligned}
$$

in $\quad$ which $\quad \Omega_{11}=-P_{1} D_{1}-D_{1} P_{1}-Y K_{1}-K_{1}^{T} Y^{T}+\tau X_{11}+X_{13}+X_{13}^{T}-F_{3} R_{3}$, $\Omega_{15}=-Y K_{3}+\tau X_{12}-X_{13}+X_{23}^{T}, \Omega_{15}=-Y K_{3}+\tau X_{12}-X_{13}+X_{23}^{T}, \Omega_{17}=P_{1} A_{1}+F_{4} R_{3}$, $\Omega_{33}=\tau X_{33}-2 P_{1}, \Omega_{37}=R_{1}-R_{2}+P_{1} A_{1}, \Omega_{55}=\tau X_{22}-X_{23}-X_{23}^{I}-F_{3} R_{4}$, then the response neural network (41) can globally asymptotically synchronize the drive neural network (40), and the

gain matrix $K$ can be designed as

$$
K=P_{1}^{-1} Y \text {. }
$$

Now, we are in a position to design a suitable sliding mode control law to guarantee the reachability of the specific switching surface.

Theorem 2 Consider the error system (4). Assume that the sliding function is given by (5) with $K=P_{1}^{-1} Y$, where $P_{1}$ and $Y$ is a feasible solution to LMIs (9) and (10). Let $\varepsilon>0$ be a constant scalar, if the sliding mode control law is designed as follows:

$$
\begin{aligned}
u(t)= & K K_{1} x(t)+K K_{2} x(t-\delta)+K K_{3} x(t-\tau(t)) \\
& +K K_{4} x(t-\sigma(t))-\rho(t) \operatorname{sgn}(S(t)),
\end{aligned}
$$

where

$$
\begin{aligned}
\rho(t)= & \left.-\varepsilon-\| D_{2}-D_{1}\right)\|\| z(t-\delta)\|-\| A_{2}\|\| g(z(t))\|-\| B_{2}\|\| g(z(t-\tau(t))) \| \\
& -\left\|C_{2}\right\| \int_{t-\sigma(t)}^{t}\|g(z(s))\| d s-\left\|A_{1}\right\|\|f(z(t))\|+\left\|B_{1}\right\|\|f(z(t-\tau(t)))\| \\
& +\left\|C_{1}\right\| \int_{t-\sigma(t)}^{t}\|f(z(s))\| d s-\left\|I_{1}(t)-I_{2}(t)\right\|,
\end{aligned}
$$

then the trajectories of the error system can be globally driven onto the sliding surface $S(t)=0$.

Proof 2 It follows from (6) and (46) that

$$
\begin{aligned}
\dot{S}(t)= & \left(D_{2}-D_{1}\right) z(t-\delta)-A_{2} g(z(t))-B_{2} g(z(t-\tau(t))) \\
& -C_{2} \int_{t-\sigma(t)}^{t} g(z(s)) d s+A_{1} f(z(t))+B_{1} f(z(t-\tau(t))) \\
& +C_{1} \int_{t-\sigma(t)}^{t} f(z(s)) d s+I_{1}(t)-I_{2}(t)+\rho(t) \operatorname{sgn}(S(t)) .
\end{aligned}
$$


Consider the following Lyapunov function as

$$
V(t)=\frac{1}{2} S^{T}(t) S(t)
$$

Calculating the time derivative of $V(t)$ along the trajectories of model (48), we obtain

$$
\begin{aligned}
\dot{V}(t)= & S^{T}(t)\left(\left(D_{2}-D_{1}\right) z(t-\delta)-A_{2} g(z(t))-B_{2} g(z(t-\tau(t)))\right. \\
& -C_{2} \int_{t-\sigma(t)}^{t} g(z(s)) d s+A_{1} f(z(t))+B_{1} f(z(t-\tau(t))) \\
& \left.+C_{1} \int_{t-\sigma(t)}^{t} f(z(s)) d s+I_{1}(t)-I_{2}(t)+\rho(t) \operatorname{sgn}(S(t))\right) .
\end{aligned}
$$

By substituting (47) into (50), and noting $S^{T}(t) \operatorname{sgn}(S(t)) \geq\|S(t)\|$, we get

$$
\dot{V}(t) \leq-\varepsilon\|S(t)\|,
$$

which means that $\dot{V}(t)<0$ for any $S(t) \neq 0$. Therefore, the trajectories of the error system (4) can be globally driven onto the sliding surface $S(t)=0$, and maintained there for all subsequent time. The proof is completed.

Remark 1 Assumption (H) was first proposed in [45]. The constants $F_{j}^{-}$and $F_{j}^{+}(i=$ $1,2, \ldots, n)$ are allowed to be positive, negative or zero. Hence, Assumption $(\mathbf{H})$ is weaker than the assumption in [30,33-35] since the boundedness and monotonicity of the activation functions are not required in this paper.

Remark 2 In [33-35], the synchronization of two nonidentical chaotic neural networks with constant delay is investigated. It is worth pointing out that the presented methods cannot be applied to analyze the synchronization of two nonidentical chaotic neural networks with time-varying delays.

\section{Example}

Example 1 Consider a two-dimensional drive neural network (1), where

$$
\begin{gathered}
f(\gamma)=\left(\tanh \left(y_{1}\right), \tanh \left(y_{2}\right)\right)^{T}, \quad I_{1}(t)=(0.9 \sin (4 t),-0.7 \cos (2 t))^{T}, \\
\delta=0.7, \quad \tau(t)=0.73|\sin t|, \quad \sigma(t)=0.2|\cos (2 t)|, \\
D_{1}=\left(\begin{array}{ll}
1 & 0 \\
0 & 1
\end{array}\right), \quad A_{1}=\left(\begin{array}{cc}
1.8 & 0.1 \\
-4.3 & 2.9
\end{array}\right), \\
B_{1}=\left(\begin{array}{ll}
-1.6 & -0.1 \\
-0.2 & -2.7
\end{array}\right), \quad C_{1}=\left(\begin{array}{cc}
-0.3 & 0.1 \\
0.1 & -0.2
\end{array}\right) .
\end{gathered}
$$

The state trajectories and phase trajectory of the neural network with initial condition $y_{1}(s)=-0.1, y_{2}(s)=0.1, s \in[-0.73,0]$ are shown in Figures 1 and 2, respectively.

The parameters of the measured output (2) are given as

$$
K_{1}=\left[\begin{array}{cc}
0.2 & 0 \\
0 & 1.3
\end{array}\right], \quad K_{2}=\left[\begin{array}{cc}
-0.1 & 0 \\
0 & 0
\end{array}\right], \quad K_{3}=\left[\begin{array}{cc}
-0.3 & 0 \\
0 & 0.1
\end{array}\right], \quad K_{4}=\left[\begin{array}{cc}
0.5 & 0 \\
0 & 0
\end{array}\right] .
$$

Assume the response neural network (3) with

$$
\begin{aligned}
& g_{1}(x)=g_{2}(x)=0.5(|x+1|-|x-1|), \quad I_{2}(t)=(1.7 \sin (3 t), 0.2 \cos (t))^{T}, \\
& D_{2}=\left(\begin{array}{cc}
1 & 0 \\
0 & 1.2
\end{array}\right), \quad A_{2}=\left(\begin{array}{cc}
6.2 & 20 \\
0.1 & 4.6
\end{array}\right),
\end{aligned}
$$

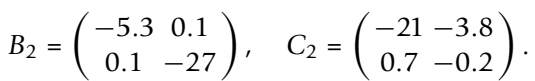




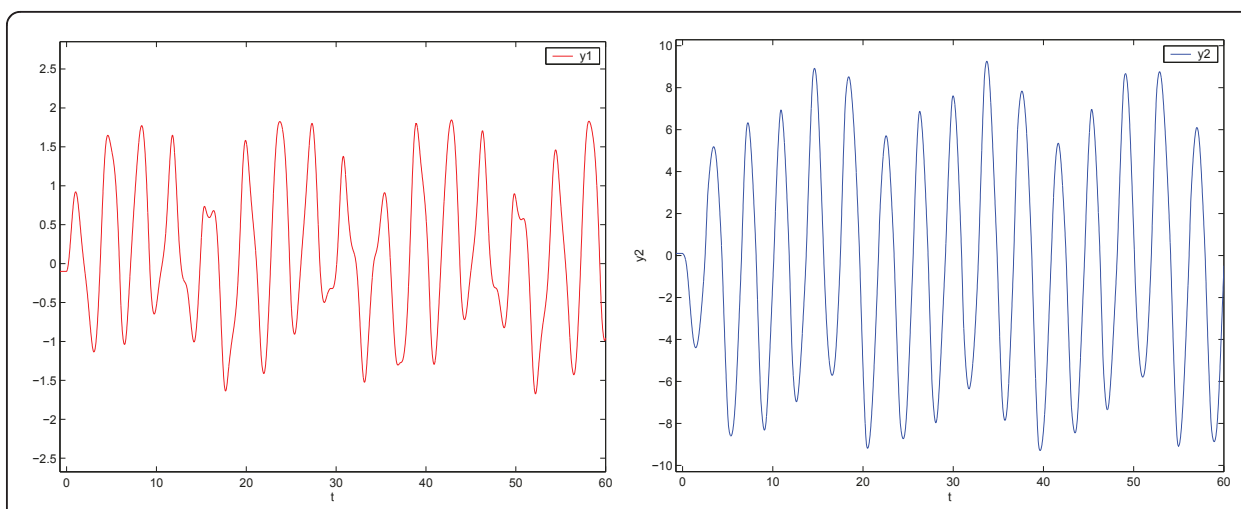

Figure 1 State trajectory of $y_{1}(t)$ and $y_{2}(t)$ of neural network (1)

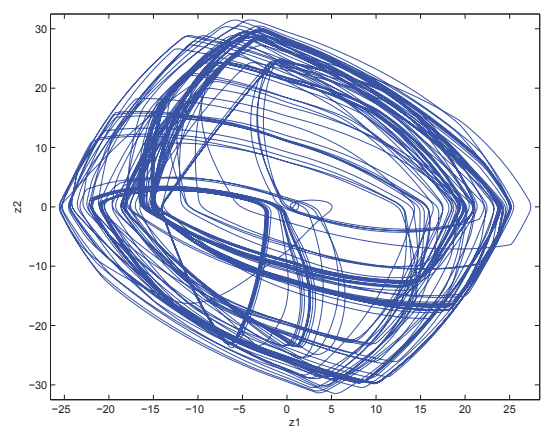

Figure 2 Phase trajectory of neural network (1).
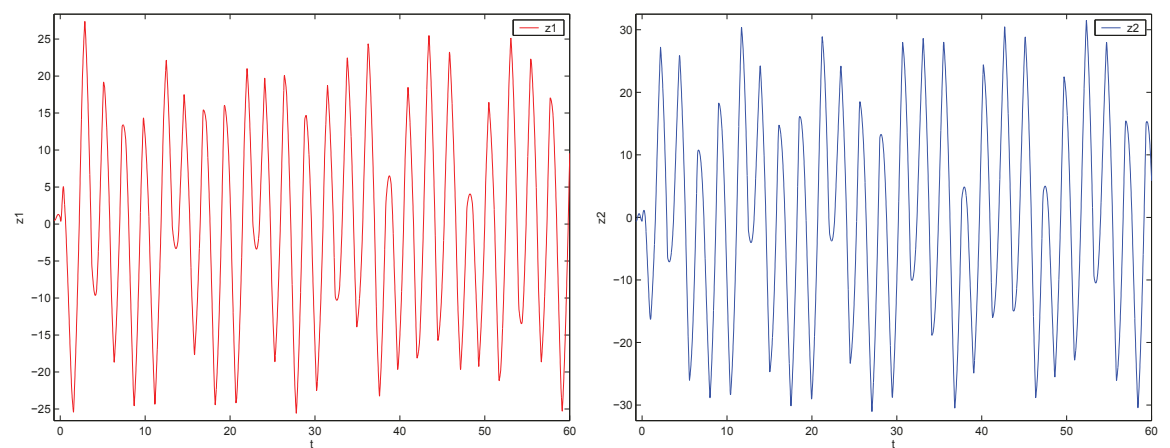

Figure 3 State trajectory of $z_{1}(t)$ and $z_{2}(t)$ of neural network (3).

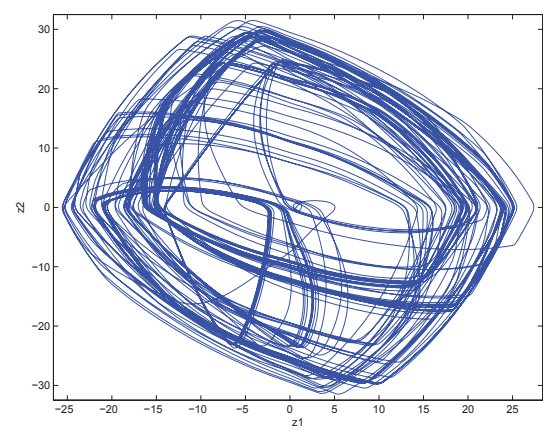

Figure 4 Phase trajectory of neural network (3) without the controller $u(t)$ 
The state trajectories and phase trajectory of the neural network with initial condition $z_{1}(s)=0.9-0.4 \sin (7 t), z_{2}(s)=-0.6 \cos (9), s \in[-0.73,0]$ are shown in Figures 3 and 4 , respectively.

It is easy to see that $\tau=0.73, \sigma=0.2$, and assumption $(\mathbf{H})$ is satisfied with $F_{1}=0, F_{2}$ $=\operatorname{diag}(1,1), F_{3}=0, F_{4}=\operatorname{diag}(0.5,0.5)$.

By the Matlab LMI Control Toolbox, we find a solution to the LMIs in (9) and (10), and obtain the gain matrix $K$ as

$$
K=\left[\begin{array}{cc}
3.6204 & -12.3204 \\
-4.9739 & 138.9582
\end{array}\right] \text {. }
$$

From Theorem 1, we know that the response neural network (3) can globally asymptotically synchronize the drive neural network (1). Figure 5 depicts the synchronization errors of state variables between drive and response systems. The numerical simulations clearly verify the effectiveness of the developed sliding mode control approach to the synchronization of nonidentical two chaotic neural networks with discrete and distributed time-varying delays as well as leakage delay.

\section{Conclusions}

In this paper, the synchronization problem has been investigated for nonidentical chaotic neural networks with discrete and distributed time-varying delays as well as leakage delay, which is more difficult and challenging than the ones for identical chaotic neural networks and nonidentical chaotic neural networks with constant delay but without leakage delay. An integral sliding mode control approach has been presented to deal with this problem. By considering a proper sliding surface and constructing LyapunovKrasovskii functional, and employing a combination of the free-weighting matrix method, Newton-Leibniz formulation and inequality technique, a sliding mode controller has been designed to achieve the asymptotical synchronization of the addressed nonidentical neural networks. Moreover, a sliding mode control law has been synthesized to guarantee the reachability of the specified sliding surface. The provided conditions are expressed in terms of LMI, and are dependent on the discrete and distributed time delays as well as leakage delay. A simulation example has been given to verify the theoretical results.

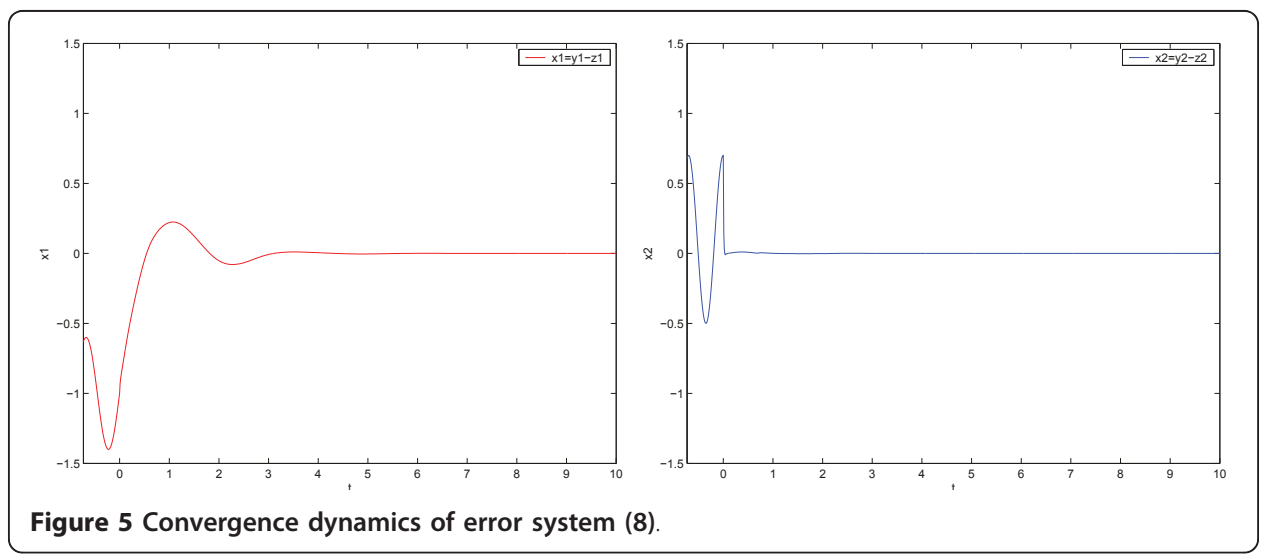


Acknowledgements

The authors would like to thank the reviewers and the editor for their valuable suggestions and comments which have led to a much improved paper. This work was supported in part by the National Natural Science Foundation of China under Grant 60974132 and 60874088.

\section{Author details}

'Department of Mathematics, Chongqing Jiaotong University, Chongqing 400074, China ${ }^{2}$ Department of Mathematics, Southeast University, Nanjing 210096, China

\section{Authors' contributions}

QS completed the main part of this paper, JC corrected the main theorems and gave the example. All authors read and approved the final manuscript.

\section{Competing interests}

The authors declare that they have no competing interests.

Received: 19 February 2011 Accepted: 23 June 2011 Published: 23 June 2011

\section{References}

1. Forti M, Nistri P, Papini D: Global exponential stability and global convergence in finite time of delayed neural networks with infinite gain. IEEE Trans Neural Netw 2005, 16:1449-1463.

2. Chen TP, Lu WL, Chen GR: Dynamical behaviors of a large class of general delayed neural networks. Neural Comput 2005, 17:949-968.

3. Park JH, Kwon OM: On improved delay-dependent criterion for global stability of bidirectional associative memory neural networks with time-varying delays. Appl Math Comput 2008, 199:435-446.

4. Ozcan N, Arik S: A new sufficient condition for global robust stability of bidirectional associative memory neural networks with multiple time delays. Nonlinear Anal Real World Appl 2009, 10:3312-3320.

5. Cao JD, Ho DWC, Huang X: LMI-based criteria for global robust stability of bidirectional associative memory networks with time delay. Nonlinear Anal 2007, 66:1558-1572

6. Xu SY, Lam J: A new approach to exponential stability analysis of neural networks with time-varying delays. Neural Netw 2006, 19:76-83.

7. Wang ZD, Liu YR, Liu XH: State estimation for jumping recurrent neural networks with discrete and distributed delays. Neural Netw 2009, 22:41-48.

8. Xu DY, Yang ZC: Impulsive delay differential inequality and stability of neural networks. J Math Anal Appl 2005, 305:107-120

9. Zeng ZG, Wang J: Improved conditions for global exponential stability of recurrent neural networks with timevarying delays. IEEE Trans Neural Netw 2006, 17:623-35

10. Wang ZS, Zhang HG, Yu W: Robust stability of Cohen-Grossberg neural networks via state transmission matrix. IEEE Trans Neural Netw 2009, 20:169-174.

11. Aihara K, Takabea T, Toyoda M: Chaotic neural networks. Phys Lett A 1990, 144:333-340.

12. Zou F, Nossek JA: A chaotic attractor with cellular neural networks. IEEE Trans Circ Syst / 1991, 38:811-822

13. Lu HT: Chaotic attractors in delayed neural networks. Phys Lett A 2002, 298:109-116.

14. Kwok T, Smith KA: A unified framework for chaotic neural-network approaches to combinatorial optimization. IEEE Trans Neural Netw 1999, 10:978-981.

15. Pecora LM, Carroll TL: Synchronization in chaotic systems. Phys Rev Lett 1990, 64:821-824

16. Yang T, Chua LO: Impulsive stabilization for control and synchronization of chaotic systems: theory and application to secure communication. IEEE Trans Circ Syst I 1997, 44:976-988.

17. Lu WL, Chen TP: Synchronization of coupled connected neural networks with delays. IEEE Trans Circ Syst I 2004, 51:2491-2503.

18. Liu YR, Wang ZD, Liu XH: On synchronization of coupled neural networks with discrete and unbounded distributed delays. Int J Comput Math 2008, 85:1299-1313.

19. Lu JQ, Ho DWC, Cao JD: Synchronization in an array of nonlinearly coupled chaotic neural networks with delay coupling. Int J Bifurc Chaos 2008, 18:3101-3111.

20. Cao JD, Li LL: Cluster synchronization in an array of hybrid coupled neural networks with delay. Neural Netw 2009, 22:335-342.

21. Yu WW, Cao JD: Synchronization control of stochastic delayed neural networks. Physica A 2007, 373:252-260.

22. Yan JJ, Lin JS, Hung ML, Liao TL: On the synchronization of neural networks containing time-varying delays and sector nonlinearity. Phys Lett A 2007, 361:70-77.

23. Park JH: Synchronization of cellular neural networks of neutral type via dynamic feedback controller. Chaos Soliton Fract 2009, 42:1299-1304.

24. Karimi HR, Maass P: Delay-range-dependent exponential $H_{\infty}$ synchronization of a class of delayed neural networks. Chaos Soliton Fract 2009, 41:1125-1135.

25. Lu JG, Chen GR: Global asymptotical synchronization of chaotic neural networks by output feedback impulsive control: an LMI approach. Chaos Soliton Fract 2009, 41:2293-2300.

26. Gao XZ, Zhong SM, Gao FY: Exponential synchronization of neural networks with time-varying delay. Nonlinear Anal 2009, 71:2003-2011

27. Tang Y, Fang JA, Miao QY: On the exponential synchronization of stochastic jumping chaotic neural networks with mixed delays and sector-bounded non-linearities. Neurocomputing 2009, 72:1694-1701.

28. Liu MQ: Optimal exponential synchronization of general chaotic delayed neural networks: an LMI approach. Neural Netw 2009, 22:949-957. 
29. Wang $\mathrm{K}$, Teng ZD, Jiang HJ: Global exponential synchronization in delayed reaction-diffusion cellular neural networks with the Dirichlet boundary conditions. Math Comput Model 2010, 52:12-24.

30. Zhang HG, Xie YH, Wang ZL, Zheng CD: Adaptive synchronization between two different chaotic neural networks with time delay. IEEE Trans Neural Netw 2007, 18:1841-1845.

31. Yoo WJ, Ji DH, Won SC: Adaptive fuzzy synchronization of two different chaotic systems with stochastic unknown parameters. Mod Phys Lett B 2010, 24:979-994.

32. Odibat ZM: Adaptive feedback control and synchronization of nonidentical chaotic fractional order systems. Nonlinear Dyn 2010, 60:479-487.

33. Huang H, Feng G: Synchronization of nonidentical chaotic neural networks with time delays. Neural Netw 2009 , 22:869-874.

34. Gan QT, Xu R, Kang XB: Synchronization of chaotic neural networks with mixed time delays. Commun Nonlinear Sci Numer Simul 2011, 16:966-974.

35. Zhang $D, X u J$ J: Projective synchronization of different chaotic time-delayed neural networks based on integral sliding mode controller. Appl Math Comput 2010, 217:164-174

36. Chen M, Jiang CS, Jiang B, Wu QX: Sliding mode synchronization controller design with neural network for uncertain chaotic systems. Chaos Soliton Fract 2009, 39:1856-1863.

37. Zhen $\mathrm{R}, \mathrm{Wu} \mathrm{XL}$, Zhang $\mathrm{JH}$ : Sliding model synchronization controller design for chaotic neural network with timevarying delay. Proceedings of the 8th World Congress on Intelligent Control and Automation 2010, 3914-3919.

38. Mei R, Wu QX, Jiang CS: Lag synchronization of delayed chaotic systems using neural network-based sliding-mode control. 2010 International Workshop on Chaos-Fractal Theory and Its Applications 2010, 3-7.

39. Gopalsamy K: Leakage delays in BAM. J Math Anal Appl 2007, 325:1117-1132.

40. Li CD, Huang TW: On the stability of nonlinear systems with leakage delay. J Franklin Inst 2009, 346:366-377.

41. Fu XL, Balasubramaniam P, Rakkiyappan R: Existence, uniqueness and stability analysis of recurrent neural networks with time delay in the leakage term under impulsive perturbations. Nonlinear Anal Real World Appl 2010, 11:4092-4108.

42. Peng SG: Global attractive periodic solutions of BAM neural networks with continuously distributed delays in the leakage terms. Nonlinear Anal Real World Appl 2010, 11:2141-2151.

43. Li XD, Cao JD: Delay-dependent stability of neural networks of neutral type with time delay in the leakage term. Nonlinearity 2010, 23:1709-1726.

44. Utkin VI: Sliding Modes in Control and Optimization. Springer, Berlin; 1992.

45. Liu YR, Wang ZD, Liu XH: Global exponential stability of generalized recurrent neural networks with discrete and distributed delays. Neural Netw 2006, 19:667-675.

doi:10.1186/1687-1847-2011-16

Cite this article as: Song and Cao: Synchronization of nonidentical chaotic neural networks with leakage delay and mixed time-varying delays. Advances in Difference Equations 2011 2011:16.

\section{Submit your manuscript to a SpringerOpen ${ }^{\odot}$ journal and benefit from:}

- Convenient online submission

- Rigorous peer review

- Immediate publication on acceptance

- Open access: articles freely available online

- High visibility within the field

- Retaining the copyright to your article

Submit your next manuscript at $>$ springeropen.com 